\title{
SEMICOMPATIBILITY AND FIXED POINT THEOREMS IN FUZZY METRIC SPACE USING IMPLICIT RELATION
}

\author{
BIJENDRA SINGH AND SHISHIR JAIN
}

Received 27 January 2005 and in revised form 29 May 2005

The concept of semicompatibility has been introduced in fuzzy metric space and it has been applied to prove results on existence of unique common fixed point of four selfmaps satisfying an implicit relation. Recently, Popa (2002) has employed a similar but not the same implicit relation to obtain a fixed point theorem for $d$-complete topological spaces. All the results of this paper are new.

\section{Introduction}

Cho et al. [2] introduced the notion of semicompatible maps in a $d$-topological space. They define a pair of self-maps $(S, T)$ to be semicompatible if conditions (i) $S y=T y$ implies that $S T y=T S y$; (ii) for sequence $\left\{x_{n}\right\}$ in $X$ and $x \in X$, whenever $\left\{S x_{n}\right\} \rightarrow$ $x,\left\{T x_{n}\right\} \rightarrow x$, then $S T x_{n} \rightarrow T x$, as $n \rightarrow \infty$, hold. However, in Fuzzy metric space (ii) implies (i), taking $x_{n}=y$ for all $n$ and $x=T y=S y$. So, we define a semicompatible pair of self-maps in fuzzy metric space by condition (ii) only. Saliga [9] and Sharma et. al [10] proved some interesting fixed point results using implicit real functions and semicompatibility in $d$-complete topological spaces. Recently, Popa in [8] used the family $F_{4}$ of implicit real functions to find the fixed points of two pairs of semicompatible maps in a $d$-complete topological space. Here, $F_{4}$ denotes the family of all real continuous functions $F:\left(\mathbb{R}^{+}\right)^{4} \rightarrow \mathbb{R}$ satisfying the following properties.

$\left(\mathrm{F}_{h}\right)$ There exists $h \geq 1$ such that for every $u \geq 0, v \geq 0$ with $F(u, v, u, v) \geq 0$ or $F(u, v, v, u) \geq 0$, we have $u \geq h v$.

$\left(\mathrm{F}_{u}\right) F(u, u, 0,0)<0$, for all $u>0$.

Jungck and Rhoades [6] (also Dhage [3]) termed a pair of self-maps to be coincidentally commuting or equivalently weak compatible if they commute at their coincidence points. This concept is most general among all the commutativity concepts in this field as every pair of commuting self-maps is $R$-weakly commuting, each pair of $R$-weakly commuting self-maps is compatible and each pair of compatible self-maps is weak compatible but the reverse is not always true. Similarly, every semicompatible pair of self-maps is weak compatible but the reverse is not true always. The main object of this paper is to obtain some fixed point theorems in the setting of fuzzy metric space using weak compatibility, 
semicompatibility, and an implicit relation. In the sequel, we derive a characterization of this implicit relation if it is in linear form and use the same for obtaining some results in fixed points. For the sake of completeness, following Kramosil and Michálek [7] and Grabiec [5], we recall some definitions and known results in fuzzy metric space.

\section{Preliminaries}

Definition 2.1. A binary operation $*:[0,1]^{2} \rightarrow[0,1]$ is called a continuous $t$-norm if $([0,1], *)$ is an abelian topological monoid with unit 1 such that $a * b \leq c * d$ whenever $a \leq c$ and $b \leq d$ for all $a, b, c$, and $d \in[0,1]$.

Examples of $t$-norm are $a * b=a b$ and $a * b=\min \{a, b\}$.

Definition 2.2 (Kramosil and Michálek [7]). The 3-tuple $(X, M, *)$ is called a fuzzy metric space if $X$ is an arbitrary set, $*$ is a continuous $t$-norm, and $M$ is a fuzzy set in $X^{2} \times[0, \infty)$ satisfying the following conditions for all $x, y, z \in X$ and $s, t>0$ :

(FM-1) $M(x, y, 0)=0$;

(FM-2) $M(x, y, t)=1$, for all $t>0$ if and only if $x=y$;

(FM-3) $M(x, y, t)=M(y, x, t)$;

(FM-4) $M(x, y, t) * M(y, z, s) \geq M(x, z, t+s)$;

$(\mathrm{FM}-5) M(x, y, \cdot):[0, \infty) \rightarrow[0,1]$ is left continuous.

Note that $M(x, y, t)$ can be thought of as the degree of nearness between $x$ and $y$ with respect to $t$. We identify $x=y$ with $M(x, y, t)=1$ for all $t>0$. The following example shows that every metric space induces a fuzzy metric space.

Example 2.3 (George and Veeramani $[4]$ ). Let $(X, d)$ be a metric space. Define $a * b=$ $\min \{a, b\}$ and for all $a, b \in X$,

$$
M(x, y, t)=\frac{t}{t+d(x, y)}, \quad \forall t>0, M(x, y, 0)=0
$$

Then $(X, M, *)$ is a fuzzy metric space. It is called the fuzzy metric space induced by the metric $d$.

Lemma 2.4 (Grabiec [5]). For all $x, y \in X, M(x, y, \cdot)$ is a nondecreasing function.

Definition 2.5 (Grabiec [5]). Let $(X, M, *)$ be a fuzzy metric space. A sequence $\left\{x_{n}\right\}$ in $X$ is said to be convergent to a point $x \in X$ if $\lim _{n \rightarrow \infty} M\left(x_{n}, x, t\right)=1$ for all $t>0$. Further, the sequence $\left\{x_{n}\right\}$ is said to be a Cauchy sequence in $X$ if $\lim _{n \rightarrow \infty} M\left(x_{n}, x_{n+p}, t\right)=1$ for all $t>0$ and $p>0$. The space is said to be complete if every Cauchy sequence in it converges to a point of it.

Remark 2.6. Since $*$ is continuous, it follows from (FM-4) that the limit of a sequence in a fuzzy metric space is unique.

In this paper, $(X, M, *)$ is considered to be the fuzzy metric space with condition (FM-6) $\lim _{t \rightarrow \infty} M(x, y, t)=1$, for all $x, y \in X$. 
Lemma 2.7 (Cho [1]). Let $\left\{y_{n}\right\}$ be a sequence in a fuzzy metric space $(X, M, *)$ with the condition (FM-6). If there exists a number $k \in(0,1)$ such that $M\left(y_{n+2}, y_{n+1}, k t\right) \geq M\left(y_{n+1}\right.$, $\left.y_{n}, t\right)$, for all $t>0$, then $\left\{y_{n}\right\}$ is a Cauchy sequence in $X$.

Lemma 2.8. Let $A$ and $B$ be two self-maps on a complete fuzzy metric space $(X, M, *)$ such that for some $k \in(0,1)$, for all $x, y \in X$ and $t>0$,

$$
M(A x, B y, k t) \geq \operatorname{Min}\{M(x, y, t), M(A x, x, t)\} .
$$

Then $A$ and $B$ have a unique common fixed point in $X$.

Proof. Let $p \in X$. Taking $x_{0}=p$, define sequence $\left\{x_{n}\right\}$ in $X$ by $A x_{2 n}=x_{2 n+1}$ and $B x_{2 n+1}=$ $x_{2 n+2}$. By taking $x=x_{2 n}, y=x_{2 n+1}$ and $x=x_{2 n}, y=x_{2 n-1}$, respectively, in the contractive condition, we obtain that

$$
M\left(x_{n+1}, x_{n}, k t\right) \geq M\left(x_{n}, x_{n-1}, t\right), \quad \forall t>0, \forall n .
$$

Therefore by Lemma 2.7, $\left\{x_{n}\right\}$ is a Cauchy sequence in $X$, which is complete. Hence, $\left\{x_{n}\right\}$ converges to some $u$ in $X$. Taking $x=x_{2 n}$ and $y=u$ and letting $n \rightarrow \infty$ in the contractive condition, we get $B u=u$. Similarly, by putting $x=u$ and $y=x_{2 n+1}$, we get $A u=u$. Therefore, $u$ is the common fixed point of the maps $A$ and $B$. The uniqueness of the common fixed point follows from the contractive condition.

Definition 2.9. Let $A$ and $S$ be mappings from a fuzzy metric space $(X, M, *)$ into itself. The mappings are said to be weak compatible if they commute at their coincidence points, that is, $A x=S x$ implies that $A S x=S A x$.

Definition 2.10. Let $A$ and $S$ be mappings from a fuzzy metric space $(X, M, *)$ into itself. Then the mappings are said to be compatible if

$$
\lim _{n \rightarrow \infty} M\left(A S x_{n}, S A x_{n}, t\right)=1, \quad \forall t>0,
$$

whenever $\left\{x_{n}\right\}$ is a sequence in $X$ such that

$$
\lim _{n \rightarrow \infty} A x_{n}=\lim _{n \rightarrow \infty} S x_{n}=x \in X .
$$

Proposition 2.11 [12]. Self-mappings $A$ and $S$ of a fuzzy metric space $(X, M, *)$ are compatible, then they are weak compatible.

The converse is not true as seen in Example 2.16.

Definition 2.12. Let $A$ and $S$ be mappings from a fuzzy metric space $(X, M, *)$ into itself. Then the mappings are said to be semicompatible if

$$
\lim _{n \rightarrow \infty} M\left(A S x_{n}, S x, t\right)=1, \quad \forall t>0,
$$

whenever $\left\{x_{n}\right\}$ is a sequence in $X$ such that

$$
\lim _{n \rightarrow \infty} A x_{n}=\lim _{n \rightarrow \infty} S x_{n}=x \in X
$$


It follows that if $(A, S)$ is semicompatible and $A y=S y$, then $A S y=S A y$. Thus if the pair $(A, S)$ is semicompatible, then it is weak compatible. The converse is not true as seen in Example 2.14.

Proposition 2.13 [13]. Let $A$ and $S$ be self-maps on a fuzzy metric space $(X, M, *)$. If $S$ is continuous, then $(A, S)$ is semicompatible if and only if $(A, S)$ is compatible.

The following is an example of a pair of self-maps $(A, S)$ which is compatible but not semicompatible. Further, it is also seen here that the semicompatibility of the pair $(A, S)$ need not imply the semicompatibility of $(S, A)$.

Example 2.14. Let $X=[0,1]$ and let $(X, M, *)$ be the fuzzy metric space with $M(x, y, t)=$ $[\exp |x-y| / t]^{-1}$, for all $x, y \in X, t>0$. Define self-map $S$ as follows:

$$
S x= \begin{cases}x & \text { if } 0 \leq x<\frac{1}{2}, \\ 1 & \text { if } x \geq \frac{1}{2} .\end{cases}
$$

Let $I$ be the identity map on $X$ and $x_{n}=1 / 2-1 / n$. Then, $\left\{I x_{n}\right\}=\left\{x_{n}\right\} \rightarrow 1 / 2$ and $\left\{S x_{n}\right\}=$ $\left\{x_{n}\right\} \rightarrow 1 / 2$. Thus, $\left\{I S x_{n}\right\}=\left\{S x_{n}\right\} \rightarrow 1 / 2 \neq S(1 / 2)$. Hence $(I, S)$ is not semicompatible. Again as $(I, S)$ is commuting, it is compatible. Further, for any sequence $\left\{x_{n}\right\}$ in $X$ such that $\left\{x_{n}\right\} \rightarrow x$ and $\left\{S x_{n}\right\} \rightarrow x$, we have $\left\{S I x_{n}\right\}=\left\{S x_{n}\right\} \rightarrow x=I x$. Hence $(S, I)$ is always semicompatible.

Remark 2.15. The above example gives an important aspect of semicompatibility as the pair of self-maps $(I, S)$ is commuting, hence it is weakly commuting, compatible, and weak compatible yet it is not semicompatible. Further, it is to be noted that the pair $(S, I)$ is semicompatible but $(I, S)$ is not semicompatible here.

The following is an example of a pair of self-maps $(A, S)$ which is semicompatible but not compatible.

Example 2.16. Let $(X, M, *)$ be a fuzzy metric space, where $X=[0,2]$, with $t$-norm defined by $a * b=\min \{a, b\}$, for all $a, b \in[0,1]$ and $M(x, y, t)=t /(t+d(x, y))$ for all $t>0$ and $M(x, y, 0)=0$, for all $x, y \in X$. Define self-maps $A$ and $S$ on $X$ as follows:

$$
A x=\left\{\begin{array}{ll}
2 & \text { if } 0 \leq x \leq 1, \\
\frac{x}{2} & \text { if } 1<x \leq 2,
\end{array} \quad S x= \begin{cases}2 & \text { if } x=1 \\
\frac{x+3}{5} & \text { otherwise }\end{cases}\right.
$$

and $x_{n}=2-1 /(2 n)$. Then we have $S(1)=A(1)=2$ and $S(2)=A(2)=1$. Also $S A(1)=$ $A S(1)=1$ and $S A(2)=A S(2)=2$. Thus $(A, S)$ is weak compatible. Again,

$$
A x_{n}=1-\frac{1}{4 n}, \quad S x_{n}=1-\frac{1}{10 n} .
$$

Thus,

$$
A x_{n} \longrightarrow 1, \quad S x_{n} \longrightarrow 1
$$

Hence $u=1$. 
Further,

$$
S A x_{n}=\frac{4}{5}-\frac{1}{20 n}, \quad A S x_{n}=2
$$

Now,

$$
\begin{gathered}
\lim _{n \rightarrow \infty} M\left(A S x_{n}, S u, t\right)=M(2,2, t)=1, \\
\lim _{n \rightarrow \infty} M\left(A S x_{n}, S A x_{n}, t\right)=\lim _{n \rightarrow \infty} M\left(2, \frac{4}{5}-\frac{1}{20 n}, t\right)=\frac{t}{t+6 / 5}<1, \quad \forall t>0 .
\end{gathered}
$$

Hence $(A, S)$ is semicompatible but it is not compatible.

For a detailed discussion of semicompatibility, we refer to $[11,13,14]$.

2.1. A class of implicit relation. Let $\Phi$ be the set of all real continuous functions $\phi$ : $\left(\mathbb{R}^{+}\right)^{4} \rightarrow \mathbb{R}$, nondecreasing in first argument and satisfying the following conditions.

(i) For $u, v \geq 0, \phi(u, v, v, u) \geq 0$ or $\phi(u, v, u, v) \geq 0$ implies that $u \geq v$.

(ii) $\phi(u, u, 1,1) \geq 0$ implies that $u \geq 1$.

Example 2.17. Define $\phi\left(t_{1}, t_{2}, t_{3}, t_{4}\right)=15 t_{1}-13 t_{2}+5 t_{3}-7 t_{4}$. Then $\phi \in \Phi$.

\section{Main results}

Theorem 3.1. Let $A, B, S$, and $T$ be self-mappings of a complete fuzzy metric space $(X, M, *)$ satisfying that

$$
A(X) \subseteq T(X), \quad B(X) \subseteq S(X) ;
$$

the pair $(A, S)$ is semicompatible and $(B, T)$ is weak compatible;

one of $A$ or $S$ is continuous;

For some $\phi \in \Phi$, there exists $k \in(0,1)$ such that for all $x, y \in X$ and $t>0$,

$$
\begin{aligned}
& \phi(M(A x, B y, k t), M(S x, T y, t), M(A x, S x, t), M(B y, T y, k t)) \geq 0, \\
& \phi(M(A x, B y, k t), M(S x, T y, t), M(A x, S x, k t), M(B y, T y, t)) \geq 0 .
\end{aligned}
$$

Then $A, B, S$, and $T$ have unique common fixed point in $X$.

Proof. Let $x_{0} \in X$ be any arbitrary point as $A(X) \subseteq T(X)$ and $B(X) \subseteq S(X)$, there exist $x_{1}, x_{2} \in X$ such that $A x_{0}=T x_{1}, B x_{1}=S x_{2}$. Inductively, construct sequences $\left\{y_{n}\right\}$ and $\left\{x_{n}\right\}$ in $X$ such that $y_{2 n+1}=A x_{2 n}=T x_{2 n+1}, y_{2 n+2}=B x_{2 n+1}=S x_{2 n+2}$, for $n=0,1,2, \ldots$ Now using (3.4) with $x=x_{2 n}, y=x_{2 n+1}$, we get

$$
\phi\left(\begin{array}{c}
M\left(A x_{2 n}, B x_{2 n+1}, k t\right), M\left(S x_{2 n}, T x_{2 n+1}, t\right), \\
M\left(A x_{2 n}, S x_{2 n}, t\right), M\left(B x_{2 n+1}, T x_{2 n+1}, k t\right)
\end{array}\right) \geq 0,
$$

that is,

$$
\phi\left(\begin{array}{c}
M\left(y_{2 n+1}, y_{2 n+2}, k t\right), M\left(y_{2 n}, y_{2 n+1}, t\right), \\
M\left(y_{2 n+1}, y_{2 n}, t\right), M\left(y_{2 n+2}, y_{2 n+1}, k t\right)
\end{array}\right) \geq 0
$$


2622 Semicompatibility, implicit relation fuzzy metric space

Using (i), we get

$$
M\left(y_{2 n+2}, y_{2 n+1}, k t\right) \geq M\left(y_{2 n+1}, y_{2 n}, t\right) .
$$

Similarly, by putting $x=x_{2 n+2}$ and $y=x_{2 n+1}$ in (3.5), we have

$$
\phi\left(\begin{array}{c}
M\left(y_{2 n+3}, y_{2 n+2}, k t\right), M\left(y_{2 n+1}, y_{2 n+2}, t\right), \\
M\left(y_{2 n+3}, y_{2 n+2}, k t\right), M\left(y_{2 n+1}, y_{2 n+2}, t\right)
\end{array}\right) \geq 0 .
$$

Using (i), we get

$$
M\left(y_{2 n+3}, y_{2 n+2}, k t\right) \geq M\left(y_{2 n+1}, y_{2 n+2}, t\right)
$$

Thus, for any $n$ and $t$, we have

$$
M\left(y_{n}, y_{n+1}, k t\right) \geq M\left(y_{n-1}, y_{n}, t\right) .
$$

Hence by Lemma 2.7, $\left\{y_{n}\right\}$ is a Cauchy sequence in $X$, which is complete. Therefore $\left\{y_{n}\right\}$ converges to $u \in X$. Its subsequences $\left\{A x_{2 n}\right\},\left\{B x_{2 n+1}\right\},\left\{S x_{2 n}\right\},\left\{T x_{2 n+1}\right\}$ also converge to $u$, that is,

$$
\begin{aligned}
& \left\{A x_{2 n}\right\} \rightarrow u, \quad\left\{B x_{2 n+1}\right\} \rightarrow u . \\
& \left\{S x_{2 n}\right\} \rightarrow u, \quad\left\{T x_{2 n+1}\right\} \rightarrow u .
\end{aligned}
$$

Case I ( $S$ is continuous). In this case, we have

$$
S A x_{2 n} \longrightarrow S u, \quad S^{2} x_{2 n} \longrightarrow S u .
$$

The semicompatibility of the pair $(A, S)$ gives

$$
\lim _{n \rightarrow \infty} A S x_{2 n}=S u
$$

Step 1. By putting $x=S x_{2 n}, y=x_{2 n+1}$ in (3.4), we obtain that

$$
\phi\left(\begin{array}{l}
M\left(A S x_{2 n}, B x_{2 n+1}, k t\right), M\left(S S x_{2 n}, T x_{2 n+1}, t\right), \\
M\left(A S x_{2 n}, S S x_{2 n}, t\right), M\left(B x_{2 n+1}, T x_{2 n+1}, k t\right)
\end{array}\right) \geq 0 .
$$

Letting $n \rightarrow \infty$, using (3.12), (3.14), (3.15), and the continuity of the $t$-norm $*$, we have

$$
\phi(M(S u, u, k t), M(S u, u, t), M(S u, S u, t), M(u, u, k t)) \geq 0,
$$

that is, $\phi(M(S u, u, k t), M(S u, u, t), 1,1) \geq 0$.

As $\phi$ is nondecreasing in the first argument, we have

$$
\phi(M(S u, u, t), M(S u, u, t), 1,1) \geq 0 .
$$

Using (ii), we get $M(S u, u, t) \geq 1$, for all $t>0$, which gives $M(S u, u, t)=1$, that is,

$$
S u=u \text {. }
$$


Step 2. By putting $x=u, y=x_{2 n+1}$ in (3.4), we obtain that

$$
\phi\left(\begin{array}{c}
M\left(A u, B x_{2 n+1}, k t\right), M\left(S u, T x_{2 n+1}, t\right), \\
M(A u, S u, t), M\left(B x_{2 n+1}, T x_{2 n+1}, k t\right)
\end{array}\right) \geq 0
$$

Taking limit as $n \rightarrow \infty$ and using (3.12) and (3.19), we get

$$
\phi(M(A u, u, k t), 1, M(A u, u, t), 1) \geq 0 .
$$

As $\phi$ is nondecreasing in the first argument, we have

$$
\phi(M(A u, u, t), 1, M(A u, u, t), 1) \geq 0
$$

Using (i), we have $M(A u, u, t) \geq 1$, for all $t>0$, which gives $u=A u$. Hence,

$$
A u=u=S u \text {. }
$$

Step 3. As $A(X) \subseteq T(X)$, there exists $w \in X$ such that $A u=S u=u=T w$. By putting $x=x_{2 n}, y=w$ in (3.4), we obtain that

$$
\phi\left(M\left(A x_{2 n}, B w, k t\right), M\left(S x_{2 n}, T w, t\right), M\left(A x_{2 n}, S x_{2 n}, t\right), M(B w, T w, k t)\right) \geq 0 .
$$

Taking limit as $n \rightarrow \infty$ and using (3.12), we get

$$
\phi(M(u, B w, k t), 1,1, M(B w, u, k t)) \geq 0 .
$$

Using (i), we have $M(u, B u, k t) \geq 1$, for all $t>0$. Hence, $M(u, B u, t)=1$. Thus, $u=B w$. Therefore $B w=T w=u$. Since $(B, T)$ is weak compatible, we get that $T B w=B T w$, that is,

$$
B u=T u
$$

Step 4. By putting $x=u, y=u$ in condition (3.4) and using (3.23) and (3.26), we obtain that

$$
\phi(M(A u, B u, k t), M(S u, T u, t), M(A u, S u, t), M(B u, T u, k t)) \geq 0,
$$

that is, $\phi(M(A u, B u, k t), M(A u, B u, t), 1,1) \geq 0$.

As $\phi$ is nondeceasing in the first argument, we have

$$
\phi(M(A u, B u, t), M(A u, B u, t), 1,1) \geq 0 .
$$

Using (ii), we have $M(A u, B u, t) \geq 1$, for all $t>0$.

Thus, $M(A u, B u, t)=1$, we have, $B u=A u$.

Therefore, $u=A u=S u=B u=T u$, that is, $u$ is a common fixed point of $A, B, S$, and $T$. Case II ( $A$ is continuous). In this case, we have

$$
A S x_{2 n} \longrightarrow A u
$$


2624 Semicompatibility, implicit relation fuzzy metric space

The semicompatibility of the pair $(A, S)$ gives

$$
A S x_{2 n} \longrightarrow S u
$$

By uniqueness of limit in fuzzy metric space, we obtain that $A u=S u$.

Step 5. By putting $x=u, y=x_{2 n+1}$ in condition (3.4), we obtain that

$$
\phi\left(\begin{array}{c}
M\left(A u, B x_{2 n+1}, k t\right), M\left(S u, T x_{2 n+1}, t\right), \\
M(A u, S u, t), M\left(B x_{2 n+1}, T x_{2 n+1}, k t\right)
\end{array}\right) \geq 0
$$

Taking limit as $n \rightarrow \infty$ and using (3.12) and (3.19), we get

$$
\phi(M(A u, u, k t), 1, M(A u, u, t), 1) \geq 0 .
$$

As $\phi$ is nondecreasing in the first argument, we have

$$
\phi(M(A u, u, t), 1, M(A u, u, t), 1) \geq 0 .
$$

Using (i), we have $M(A u, u, t) \geq 1$, for all $t>0$, which gives $u=A u$ and the rest of the proof follows from Step 3 onwards of the previous case.

Uniqueness. Let $z$ be another common fixed point of $A, B, S$, and $T$.

Then $z=A z=B z=S z=T z$.

Putting $x=u$ and $y=z$ in (3.4), we get

$$
\phi(M(A u, B z, k t), M(S u, T z, t), M(A u, S u, t), M(B z, T z, k t)) \geq 0,
$$

that is, $\phi(M(u, z, k t), M(u, z, t), 1,1) \geq 0$.

As $\phi$ is nondecreasing in the first argument, we have

$$
\phi(M(u, z, t), M(u, z, t), 1,1) \geq 0 \text {. }
$$

Using (i), we have $M(u, z, t) \geq 1$, for all $t>0$.

Hence $M(u, z, t)=1$, that is, $u=z$. Therefore, $u$ is the unique common fixed point of the self-maps $A, B, S$, and $T$.

Corollary 3.2. Let $A, B, S$, and $T$ be self-mappings of a complete fuzzy metric space $(X, M$, *) satisfying conditions (3.1), (3.4),(3.5), and that

$$
\begin{aligned}
& \text { the pairs }(A, S) \text { and }(B, T) \text { are semicompatible; } \\
& \text { one of } A, B, S \text {, or } T \text { is continuous. }
\end{aligned}
$$

Then $A, B, S$, and $T$ have a unique common fixed point in $X$.

Proof. As semicompatibility implies weak compatibility, the proof follows from Theorem 3.1.

On taking $A=B$ in Theorem 3.1, we have the following corollary. 
Corollary 3.3. Let $A, S$, and $T$ be self-mappings of a complete fuzzy metric space $(X, M, *)$ satisfying that

$$
A(X) \subseteq T(X) \cap S(X) ;
$$

the pair $(A, S)$ is semicompatible and $(A, T)$ is weak compatible;

one of $A$ or $S$ is continuous.

For some $\phi \in \Phi$, there exists $k \in(0,1)$ such that for all $x, y \in X$ and $t>0$,

$$
\begin{aligned}
& \phi(M(A x, A y, k t), M(S x, T y, t), M(A x, S x, t), M(A y, T y, k t)) \geq 0, \\
& \phi(M(A x, A y, k t), M(S x, T y, t), M(A x, S x, k t), M(A y, T y, t)) \geq 0 .
\end{aligned}
$$

Then $A, S$, and $T$ have a unique common fixed point in $X$.

Now, taking $S=I$ and $T=I$ in Theorem 3.1, the conditions (3.1), (3.2), (3.3) are satisfied trivially, and we get the following corollary.

Corollary 3.4. Let $A$ and $B$ be self-mappings of a complete fuzzy metric space $(X, M, *)$ such that for some $\phi \in \Phi$, there exists some $k \in(0,1)$ such that for all $x, y \in X$ and for all $t>0$,

$$
\begin{aligned}
& \phi(M(A x, B y, k t), M(x, y, t), M(A x, x, t), M(B y, y, k t)) \geq 0, \\
& \phi(M(A x, B y, k t), M(x, y, t), M(A x, x, k t), M(B y, y, t)) \geq 0 .
\end{aligned}
$$

Then $A$ and $B$ have a unique common fixed point in $X$.

Theorem 3.5. Let $A, B, S$, and $T$ be self-mappings of a complete fuzzy metric space $(X, M, *)$ satisfying conditions (3.1), (3.3), (3.4), (3.5), and that

$$
\text { the pair }(A, S) \text { is compatible and }(B, T) \text { is weak compatible. }
$$

Then $A, B, S$, and T have a unique common fixed point in $X$.

Proof. In view of Proposition 2.13 and Theorem 3.1, it suffices to prove the result when $A$ is continuous. As in the proof of Theorem 3.1, the sequence $\left\{y_{n}\right\} \rightarrow u \in X$ and (3.12) and (3.13) are satisfied. As $A$ is continuous, we have

$$
A S x_{2 n} \longrightarrow A u, \quad A A x_{2 n} \longrightarrow A u
$$

The compatibility of $(A, S)$ gives

$$
\lim _{n \rightarrow \infty} A S x_{2 n}=A u=\lim _{n \rightarrow \infty} S A x_{2 n} .
$$

Step I. By putting $x=A x_{2 n}$ and $y=x_{2 n+1}$ in condition (3.4), we get that

$$
\phi\left(\begin{array}{c}
M\left(A A x_{2 n}, B x_{2 n+1}, k t\right), M\left(S A x_{2 n}, T x_{2 n+1}, t\right), \\
M\left(A A x_{2 n}, S A x_{2 n}, t\right), M\left(B x_{2 n+1}, T x_{2 n+1}, k t\right)
\end{array}\right) \geq 0 .
$$


2626 Semicompatibility, implicit relation fuzzy metric space

Letting $n \rightarrow \infty$, using (3.12) and (3.13), we obtain that

$$
\phi(M(A u, u, k t), M(A u, u, t), M(A u, A u, t), M(u, u, k t)) \geq 0,
$$

that is, $\phi(M(A u, u, k t), M(A u, u, t), 1,1) \geq 0$, that is, $\phi(M(A u, u, t), M(A u, u, t), 1,1) \geq 0$.

Using (ii), we have $M(A u, u, t) \geq 1, t>0$. Therefore $u=A u$.

Step II. As $A(X) \subseteq T(X)$, there exists $w \in X$ such that $A u=S u=u=T w$. By putting $x=x_{2 n}, y=w$ in (3.4), we obtain that

$$
\phi\left(M\left(A x_{2 n}, B w, k t\right), M\left(S x_{2 n}, T w, t\right), M\left(A x_{2 n}, S x_{2 n}, t\right), M(B w, T w, k t)\right) \geq 0 .
$$

Taking limit as $n \rightarrow \infty$ and using (3.12), we get

$$
\phi(M(u, B w, k t), 1,1, M(B w, u, k t)) \geq 0 .
$$

Using (i), we have $M(u, B u, k t) \geq 1$, for all $t>0$. Hence, $M(u, B u, t)=1$. Thus, $u=B w$. Therefore $B w=T w=u$. As $(B, T)$ is weak compatible, we have $T B w=B T w$, that is, $B u=T u$.

Step III. Again as $u=B w$ and $B(X) \subseteq S(X)$, there exists $v \in X$ such that $u=B w=S v$. By putting $x=v, y=w$ in (3.4), we have

$$
\phi(M(A v, B w, k t), M(S v, T w, t), M(A v, S v, t), M(B w, T w, k t)) \geq 0
$$

that is, $\phi(M(A v, S v, k t), 1, M(A v, S v, t), 1) \geq 0$, that is, $\phi(M(A v, S v, t), 1, M(A v, S v, t), 1) \geq$ 0 .

Using (i), we have $M(A v, S v, t) \geq 1$, for all $t>0$.

This gives $A v=S v$. As $(A, S)$ is compatible, we have $A S v=S A v$ or $A u=S u=u$. Also $A u=B u$ follows from Step 4 in the proof of Theorem 3.1 and it follows that $u$ is a common fixed point of the four maps $A, B, S$, and $T$. The uniqueness follows as in the proof of Theorem 3.1.

Corollary 3.6. Let $A, B, S$, and T be self-mappings of a complete fuzzy metric space $(X, M$, *) satisfying conditions (3.1), (3.4), (3.5), and that

$$
\begin{aligned}
& \text { the pairs }(A, S) \text { and }(B, T) \text { are compatible; } \\
& \text { one of } A, B, S \text {, or } T \text { is continuous. }
\end{aligned}
$$

Then $A, B, S$, and $T$ have a unique common fixed point in $X$.

Proof. As compatibility implies weak compatibility, the proof follows from Theorem 3.5.

If we take $A=I$, the identity map on $X$ in Theorem 3.5, we have the following result for three self-maps, none of which is continuous and just a pair of them is needed to be weak compatible. 
Corollary 3.7. Let $B, S$, and $T$ be self-mappings of a complete fuzzy metric space $(X, M, *)$, satisfying that

the pair $(B, T)$ is weak compatible;

$T$ is surjective.

For some $\phi \in \Phi$, there exists $k \in(0,1)$ such that for all $x, y \in X$ and $t>0$,

$$
\begin{aligned}
& \phi(M(x, B y, k t), M(S x, T y, t), M(x, S x, t), M(B y, T y, k t)) \geq 0, \\
& \phi(M(x, B y, k t), M(S x, T y, t), M(x, S x, k t), M(B y, T y, t)) \geq 0 .
\end{aligned}
$$

Then $B, S$, and $T$ have a unique common fixed point in $X$.

Now, taking $A=I$ and $B=I$ in Corollary 3.6, the condition (3.49) is satisfied trivially and we get an important result for surjective maps as follows.

Corollary 3.8. Let $S$ and $T$ be two surjective self-mappings of a complete fuzzy metric space $(X, M, *)$ such that for some $\phi \in \Phi$, there exists some $k \in(0,1)$ satisfying

$$
\begin{aligned}
& \phi(M(x, y, k t), M(S x, T y, t), M(x, S x, t), M(y, T y, k t)) \geq 0, \\
& \phi(M(x, y, k t), M(S x, T y, t), M(x, S x, k t), M(y, T y, t)) \geq 0,
\end{aligned}
$$

for all $x, y \in X$ and for all $t>0$.

Then $S$ and $T$ have a unique common fixed point in $X$.

3.1. A characterization of $\Phi$ in linear form. Define $\phi\left(t_{1}, t_{2}, t_{3}, t_{4}\right)=a t_{1}+b t_{2}+c t_{3}+d t_{4}$, where $a, b, c, d \in \mathbb{R}$ with $a+b+c+d=0, a>0, a+c>0, a+b>0$, and $a+d>0$. Then $\phi \in \Phi$.

Proof. For $u, v \geq 0$ and $\phi(u, v, v, u) \geq 0$, we have

$$
(a+d) u+(b+c) v \geq 0
$$

that is, $(a+d) u \geq(a+d) v$. Hence $u \geq v$, since $a+d>0$.

Again,

$$
\phi(u, v, u, v) \geq 0
$$

gives

$$
(a+c) u+(b+d) v \geq 0
$$

that is, $(a+c) u-(a+c) v \geq 0$. Hence, $u \geq v$ as $(a+c)>0$.

Also, $\phi(u, u, 1,1) \geq 0$ gives

$$
(a+b) u+(c+d) \geq 0,
$$

that is, $(a+b) u \geq-(c+d)$, that is, $(a+b) u \geq(a+b)$, as $a+b+c+d=0$. Hence, $u \geq 1$.

As $a>0, \phi$ is nondecreasing in the first argument and the result follows. 
2628 Semicompatibility, implicit relation fuzzy metric space

Corollary 3.9. Let $A$ and $B$ be self-mappings of a complete fuzzy metric space $(X, M, *)$ such that there exists some $k \in(0,1)$ satisfying

$$
\begin{aligned}
& a M(A x, B y, k t)+b M(x, y, t)+c M(A x, x, t)+d M(B y, y, k t) \geq 0, \\
& a M(A x, B y, k t)+b M(x, y, t)+c M(A x, x, k t)+d M(B y, y, t) \geq 0,
\end{aligned}
$$

for all $x, y \in X$, for all $t>0$, and for some fixed $a, b, c, d \in \mathbb{R}$ such that $a>0, a+b>0$, $a+c>0, a+d>0$, and $a+b+c+d=0$.

Then $A$ and $B$ have a unique common fixed point in $X$.

Proof. Using the characterization of $\Phi$ in Corollary 3.4, the result follows.

Corollary 3.10. Let $A$ and $B$ be self-mappings of a complete fuzzy metric space $(X, M, *)$ such that there exists some $k \in(0,1)$ satisfying

$$
M(A x, B y, k t) \geq b_{0} M(x, y, t)+c_{0} M(A x, x, t), \quad \forall x, y \in X, \forall t>0,
$$

where $b_{0}, c_{0} \in(0,1)$ with $b_{0}+c_{0}=1$. Then $A$ and $B$ have $a$ unique common fixed point in $X$. Proof. Choosing $a=1, d=0, b=-b_{0}$, and $c=-c_{0}, c_{0}>0$, in Corollary 3.9 and using the fact that $M(x, y, \cdot)$ is a nondecreasing function, the second condition of Corollary 3.9 is trivially satisfied and the result follows.

Combining Corollary 3.10 and Lemma 2.8, we have the following important result.

Corollary 3.11. Let $A$ and $B$ be self-mappings of a complete fuzzy metric space $(X, M, *)$ such that there exists some $k \in(0,1)$ satisfying

$$
M(A x, B y, k t) \geq b_{0} M(x, y, t)+c_{0} M(A x, x, t), \quad \forall x, y \in X, \forall t>0,
$$

where $b_{0}, c_{0} \in[0,1]$ with $b_{0}+c_{0}=1$. Then $A$ and $B$ have a unique common fixed point in $X$. Corollary 3.12. Let $A$ be self-mapping of a complete fuzzy metric space $(X, M, *)$ such that there exists some $k \in(0,1)$ satisfying

$$
M(A x, A y, k t) \geq b_{0} M(x, y, t)+c_{0} M(A x, x, t), \quad \forall x, y \in X, \forall t>0,
$$

where $b_{0}, c_{0} \in[0,1]$ with $b_{0}+c_{0}=1$. Then $A$ has a unique fixed point in $X$.

Proof. Taking $A=B$ in Corollary 3.11, the result follows.

Remark 3.13. If we take $b_{0}=1$ and $c_{0}=0$ in Corollary 3.12, we get the Banach contraction principle in the setting of fuzzy metric space as given by Grabiec in [5].

\section{Acknowledgment}

Authors thank Shobha Jain, Department of Mathematics, MB Khalsa College, Devi Ahilya University, Indore, for her cooperation in the preparation of this paper. 


\section{References}

[1] Y. J. Cho, Fixed points in fuzzy metric spaces, J. Fuzzy Math. 5 (1997), no. 4, 949-962.

[2] Y. J. Cho, B. K. Sharma, and D. R. Sahu, Semi-compatibility and fixed points, Math. Japon. 42 (1995), no. 1, 91-98.

[3] B. C. Dhage, On common fixed points of pairs of coincidentally commuting mappings in D-metric spaces, Indian J. Pure Appl. Math. 30 (1999), no. 4, 395-406.

[4] A. George and P. Veeramani, On some results in fuzzy metric spaces, Fuzzy Sets and Systems 64 (1994), no. 3, 395-399.

[5] M. Grabiec, Fixed points in fuzzy metric spaces, Fuzzy Sets and Systems 27 (1988), no. 3, 385389.

[6] G. Jungck and B. E. Rhoades, Fixed points for set valued functions without continuity, Indian J. Pure Appl. Math. 29 (1998), no. 3, 227-238.

[7] I. Kramosil and J. Michálek, Fuzzy metrics and statistical metric spaces, Kybernetika (Prague) 11 (1975), no. 5, 336-344.

[8] V. Popa, Fixed points for non-surjective expansion mappings satisfying an implicit relation, Bul. Ştiinţ. Univ. Baia Mare Ser. B Fasc. Mat.-Inform. 18 (2002), no. 1, 105-108.

[9] L. M. Saliga, Fixed point theorems for non-self maps in d-complete topological spaces, Int. J. Math. Math. Sci. 19 (1996), no. 1, 103-110.

[10] B. K. Sharma, D. R. Sahu, M. Bounias, and A. Bonaly, Fixed points for non-surjective expansion mappings, Int. J. Math. Math. Sci. 21 (1998), no. 2, 277-288.

[11] B. Singh and S. Jain, Semi-compatibility and fixed point theorems in Menger space, Journal of the Chungcheong Mathematical Society 17 (2004), no. 1, 1-17.

[12] - A fixed point theorem in Menger space through weak compatibility, J. Math. Anal. Appl. 301 (2005), no. 2, 439-448.

[13] Semi-compatibility, compatibility and fixed point theorems in fuzzy metric space, Journal of the Chungcheong Mathematical Society 18 (2005), no. 1, 1-23.

[14] B. Singh, S. Jain, and S. Jain, Semi-compatibility and fixed point theorems in an unbounded Dmetric space, Int. J. Math. Math. Sci. 2005 (2005), no. 5, 789-801.

Bijendra Singh: School of Studies in Mathematics, Vikram University, University Road Ujjain, 456010 Madhya Pradesh, India

E-mail address: bijendrasingh@yahoo.com

Shishir Jain: Shri Vaishnav Institute of Technology and Science, Indore 453331, Madhya Pradesh, India

E-mail address: jainshishir11@rediffmail.com 


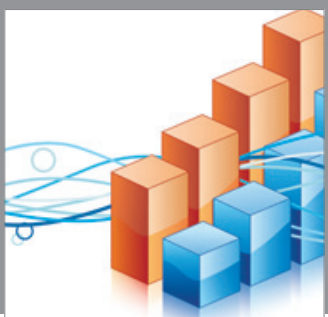

Advances in

Operations Research

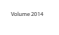

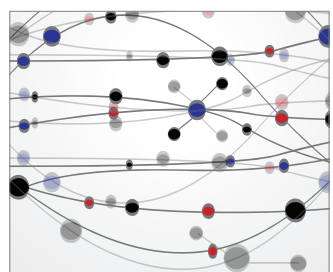

\section{The Scientific} World Journal
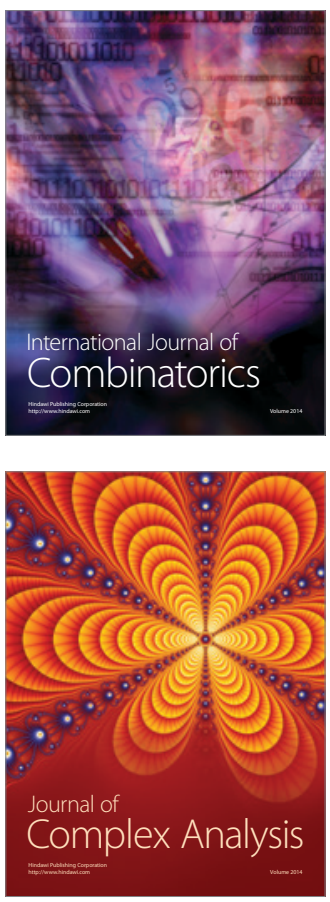

International Journal of

Mathematics and

Mathematical

Sciences
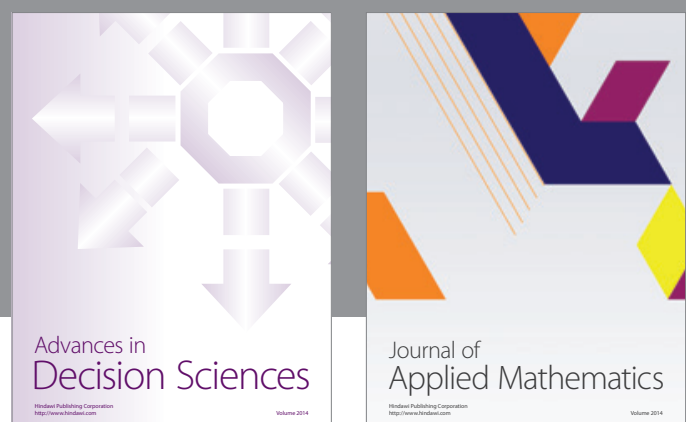

Journal of

Applied Mathematics
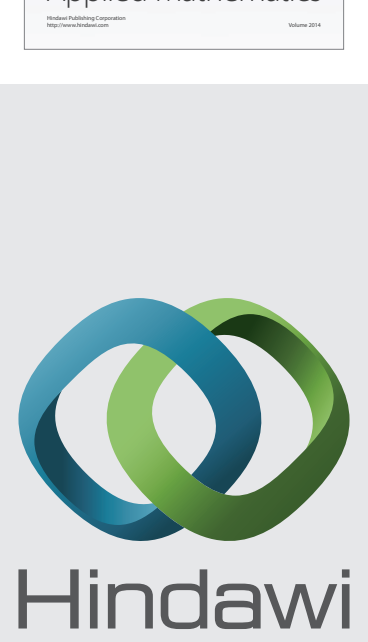

Submit your manuscripts at http://www.hindawi.com
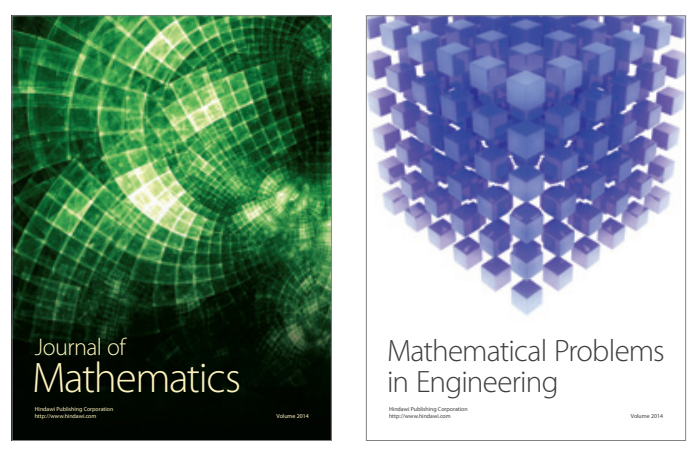

Mathematical Problems in Engineering
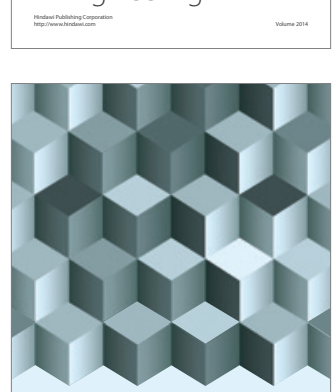

Journal of

Function Spaces
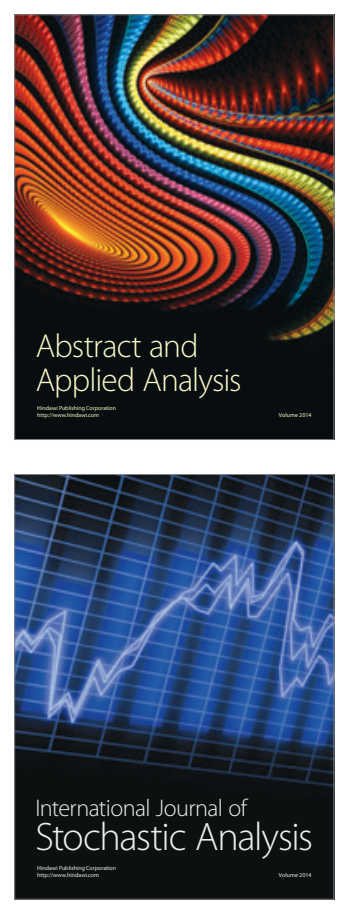

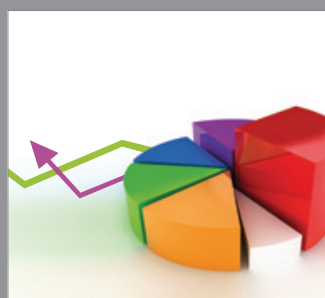

ournal of

Probability and Statistics

Promensencen
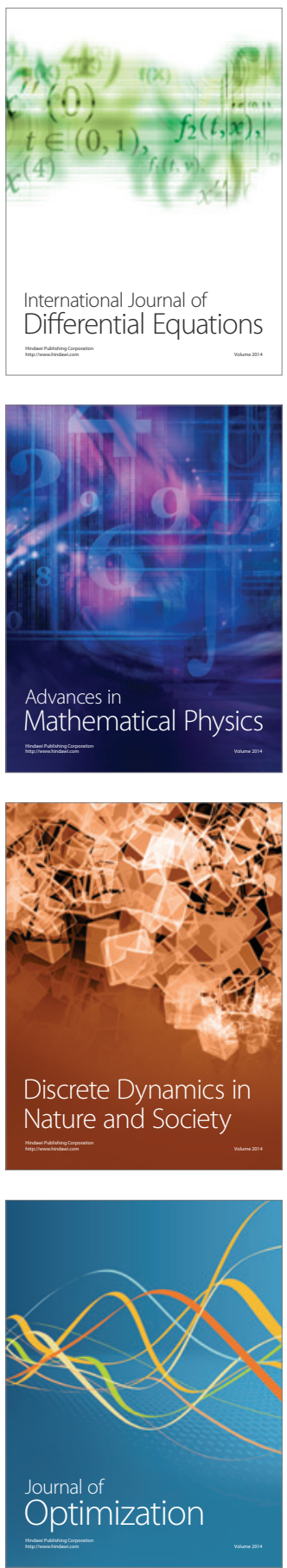